\title{
EPIDEMIOLOGIA MOLECOLARE DI CEPPI DI STENOTROPHOMONAS MALTOPHILIA ISOLATI DA PAZIENTI CON FIBROSI CISTICA
}

\author{
Gherardi G., Fiscarelli E., Dicuonzo G . \\ Centro Fibrosi Cistica, Ospedale Pediatrico Bambin Gesù, Roma; \\ Dipartimento Patologia Clinica, Laboratorio di Microbiologia, \\ Università Campus Bio-medico, Roma.
}

Introduzione. Lo Stenotrophomonas maltophilia (SM) è un bacillo gram negativo non fermentante ad elevata diffusione ambientale. Importante patogeno opportunista in pazienti immunocompromessi. Negli ultimi anni è stato sempre con maggiore frequenza isolato in pazienti affetti da fibrosi cistica, dove il suo ruolo di colonizzatore o agente infettante è ancora a poco chiaro. Importante nelle infezioni ospedaliere, ancora molto poco si conosce sulla sua epidemiologia.

Metodi. 89 ceppi di SM isolati dal 1 gennaio 2003 al 31 marzo 2004 da campioni delle vie aeree di 42 pazienti affetti da fibrosi cistica dell' Ospedale Pediatrico Bambin Gesù sono stati genotipizzati mediante PFGE.

Risultati. 39 tipi di PFGE sono stati trovati, di cui 21 rappresentati da isolati multipli, 18 da isolati singoli. Sei tipi di PFGE erano formati da più sottotipi. I tipi di PFGE più rappresentati erano il tipo 4 ( 11 isolati), i tipi 9 e 11 ( 7 isolati ciascuno). La maggior parte dei ceppi isolati dallo stesso paziente erano tra loro correlati geneticamente. Sono stati ritrovati profili di PFGE unici per isolati provenienti dallo stesso paziente (es. PFGE tipo 3, 6, 9, 10, 25). Sono stati anche osservati casi di isolati clinici di SM geneticamente correlati provenienti da pazienti diversi (es. PFGE tipo 4 e 11). Sono stati peraltro trovati 6 casi di pazienti, dove ceppi di SM, provenienti dallo stesso paziente, presentavano profili di PFGE non correlati (paziente B, F, N, S, AG, AJ).

Conclusioni. E' stata osservata alta variabilità tra $\mathrm{i}$ ceppi di S. maltophilia genotipizzati. E' stata confermata in vari casi la persistenza dello stesso clone tra ceppi isolati dallo stesso paziente in tempi diversi. Sono stati osservati diversi piccoli clusters sospetti di diffusione clonale orizzontale rappresentati da ceppi con identico profilo di PFGE isolati in pazienti diversi a sostegno di una trasmissione persona-persona. 